\title{
Population Genetic Analyses of Fusarium asiaticum Populations from Barley Suggest a Recent Shift Favoring 3ADON Producers in Southern China
}

\author{
H. Zhang, Z. Zhang, T. van der Lee, W. Q. Chen, J. Xu, \\ J. S. Xu, L. Yang, D. Yu, C. Waalwijk, and J. Feng
}

First, second, forth, fifth, sixth, and tenth authors: State Key Laboratory for Biology of Plant Diseases and Insect Pests, Institute of Plant Protection, Chinese Academy of Agriculture Sciences, Beijing 100193, China; third and ninth authors: Plant Research International BV, P.O. Box 166700 AA, The Netherlands; and seventh and eighth authors: Institute for Plant Protection and Soil Sciences, Hubei Academy of Agricultural Sciences, 430064, Wuhan, China.

Accepted for publication 15 November 2009.

\begin{abstract}
Zhang, H., Zhang, Z., van der Lee, T., Chen, W. Q., Xu, J., Xu, J. S., Yang, L., Yu, D., Waalwijk, C., and Feng, J. 2010. Population genetic analyses of Fusarium asiaticum populations from barley suggest a recent shift favoring $3 \mathrm{ADON}$ producers in southern China. Phytopathology 100:328-336.

Fusarium asiaticum is the predominant causal agent of Fusarium head blight (FHB) in southern China. The genetic diversity was assessed by analyzing 448 single-spore $F$. asiaticum isolates from 18 sampling sites that were 10 to $2,000 \mathrm{~km}$ apart, using seven highly informative variable number of tandem repeat (VNTR) markers. This analysis showed a

this genetic population subdivision and the mycotoxin produced. Our results show that the dramatic cline in trichothecene chemotypes may be explained by a recent and significant invasion of 3-acetyldeoxynivalenol ( $3 \mathrm{ADON})$ producers in FHB pathogen composition in the middle valley. Using Bayesian statistics, we found a biased gene flow from $3 \mathrm{ADON}$ to nivalenol (NIV) populations. In addition, we observed significant genetic differentiation and linkage disequilibrium between NIV- and 3ADONproducing isolates at the same sampling sites. The impact of the changed agronomy and trade of cereal commodities on the spread of the new Fusarium population and the consequent increase of FHB observed in southern China are discussed.
\end{abstract} significant degree of population subdivision $(P<0.001)$ among populations from upper, middle, and lower valleys of the Yangtze River, with little gene flow $(\mathrm{Nm}=1.210)$. We observed a strong association between
Additional keywords: genetic variation.
Fusarium head blight (FHB) or scab is an economically devastating disease of wheat, barley, and other small cereal grains all over the world $(16,36)$. The disease is a growing threat to the world's food supply due to recent outbreaks in Asia, Europe, and South America (14). In addition to quantitative yield losses, harvested grain sustains qualitative problems of contamination with mycotoxins such as nivalenol (NIV), deoxynivalenol (DON), and several acetylated derivatives of NIV and DON (20). These trichothecenes pose a significant risk to food safety and animal health because they inhibit eukaryotic protein synthesis and modify immune function $(14,31)$.

In China, the first outbreak of FHB was reported in 1936, when the percentage of scabbed spikes was as high as $95 \%$ in cv. Jinda 2905 in Xuancheng County, Anhui Province (38). Since then, the disease occurred frequently in the middle and downstream regions of the Yangtze River, including the provinces of Jiangsu, Zhejiang, Anhui, and Hubei, and in the northeastern region, including Heilongjiang Province. During the last few years, an increasing number of wheat-growing regions throughout the country,

Corresponding author: J. Feng; E-mail address: Jfeng@ippcaas.cn

* The $\boldsymbol{e}$-Xtra logo stands for "electronic extra" and indicates that the online version contains three tables showing the results of seven VNTR markers on $448 \mathrm{~F}$. asiaticum isolates, the reference strains used to validate the newly designed chemotype-specific PCR primers, and Nei's unbiased genetic distance based on the VNTR data and geographical distance for the $F$. asiaticum strains from 18 sampling sites.

doi:10.1094/PHYTO-100-4-0328

(C) 2010 The American Phytopathological Society including the provinces of Henan, Shaanxi, Ningxia, Gansu, Qinghai, Hebei, Shandong, and Sichuan, have been affected by the disease, resulting in extensive yield losses in wheat and barley. This indicates that FHB epidemics are becoming more frequent, more severe, and more widespread in China.

FHB is caused by a complex of Fusarium graminearum species, which consists of at least 12 phylogenetically distinct species, most of which cannot be distinguished morphologically (23-25,29). Previous studies indicated that Chinese strains were mainly divided into two groups (e.g., F. graminearum sensu stricto and $F$. asiaticum) $(26,39) . F$. graminearum sensu stricto mainly existed in the cooler wheat regions where the annual average temperature was $\leq 15^{\circ} \mathrm{C}$. The vast majority of $F$. asiaticum isolates were found in the warmer regions, where the annual average temperature is $>15^{\circ} \mathrm{C}$ and where FHB epidemics occur most frequently (26). Yang et al. (39) screened a large number of FHB isolates collected from barley in 23 counties of seven provinces and two municipalities along the Yangtze River in southern China. F. asiaticum was the predominant species in all regions whereas, in the upper valleys of the Yangtze River, $F$. graminearum sensu stricto and $F$. meridionale were also found.

For population studies, random amplified DNA polymorphism (RAPD) and amplified fragment length polymorphism (AFLP) have been used to analyze the genetic variation and biogeographic structure of $F$. graminearum complex on wheat in China $(12,26)$. However, there is no report about the genetic structure of $F$. asiaticum, the predominant pathogen on barley in China. Compared with RAPD and AFLP, variable number of tandem repeat (VNTR) markers are more desirable for population genetic 
analysis due to the simplicity of collecting accurate polymorphic data and due to their co-dominance (30). Generally, developing VNTR markers is costly and cumbersome; therefore, few VNTR markers have been reported for $F$. graminearum (13). However, the whole-genome sequence of $F$. graminearum strain $\mathrm{PH}-1$ (NRRL 31084) made it convenient to design new VNTRs $(30,32)$.

Type B trichothecene-producing species of the FHB complex consist of members of the $F$. graminearum species complex (FGSC) and five closely related species. These species have the tendency to produce different, strain-specific trichothecenes $(19,25)$ : NIV and acetylated derivatives (NIV chemotype), DON and primarily 3-acetyldeoxynivalenol (3ADON chemotype), or DON and primarily 15-acetyldeoxynivalenol (15ADON chemotype). These chemotypes may affect species or population ecology because oxygenation or acetylation is known to alter the toxicity and bioactivity of tricothecenes $(2,17)$. It has been hypothesized that trichothecene chemotype polymorphisms are maintained by balancing selection, suggesting that chemotype differences have fitness consequences $(18,34)$.. According to sequences of some vital genes in the trichothecene biosynthesis pathway, a number of polymerase chain reaction (PCR) assays have been developed that allow the detection and differentiation of $3 \mathrm{ADON}, 15 \mathrm{ADON}$, and NIV chemotypes among type B trichothecene-producing species $(6,16,33)$. In previous studies, all three trichothecene chemotypes have been found in $F$. asiaticum in China (41). Recently, we reported that the distribution of type B trichothecene producers on barley showed a dramatic gradient along the Yangtze River (39). DON producers dominate the lower valleys while NIV producers are dominant in the upper valleys. Therefore, we hypothesize that DON-producing isolates were replacing NIV producers from east to west (39). However, additional evidence is needed to test this hypothesis. Ward et al. (35) demonstrated that the population structure of $F$. graminearum in Canada was strongly associated with chemotype. How- ever, the association between trichothecene chemotype and population subdivision among $F$. asiaticum from China is still unknown.

In this study, we (i) investigated the population structure of $F$. asiaticum collected from the upper, middle, and lower reaches of the Yangtze River using VNTR markers and (ii) developed a effective set of diagnostic primers based on single-nucleotide polymorphisms (SNPs) among three different chemotype isolates and determined the distribution of two acetylated derivatives of DON (15ADON and 3ADON) in southern China. Collectively, the results of this study provide critical information on the structure and distribution of genotypes and the mycotoxin diversity within $F$. asiaticum, which is needed to develop effective FHB management practices in the field.

\section{MATERIALS AND METHODS}

Biological materials. The 448 single-spore strains were isolated from barley spikes with FHB symptoms, which were collected from 18 counties in seven provinces and two municipalities along the Yangtze River (Fig. 1). The details of the isolate, such as the number of strains tested in each county, were summarized in Table 1. Methods for culturing strains, DNA isolation, and identification at species level by species-specific PCR have been described previously (39).

VNTR analysis. Genotyping of the isolates was performed by VNTR-PCR with seven pairs of VNTR primers ( $e$-Xtra) designed by Suga et al. (30). The reaction mixture was $20 \mu \mathrm{l}$ containing $10 \mathrm{mM}$ Tris- $\mathrm{HCl}$ (pH 8.3), $50 \mathrm{mM} \mathrm{KCl}, 1.5 \mathrm{mM} \mathrm{MgCl} 2,200 \mu \mathrm{M}$ dNTP, $1 \mu \mathrm{m}$ of each primer, 0.5 unit of Taq polymerase (Takara, Japan), and $10 \mathrm{ng}$ of genomic DNA. PCR was carried out in a Mastercycler gradient (Eppendorf, Germany), using the following cycling parameters: $94^{\circ} \mathrm{C}$ for $1 \mathrm{~min}$; then, 30 cycles of $95^{\circ} \mathrm{C}$ for $30 \mathrm{~s}, 58^{\circ} \mathrm{C}$ for $30 \mathrm{~s}$, and $72^{\circ} \mathrm{C}$ for $30 \mathrm{~s}$; and a final extension at

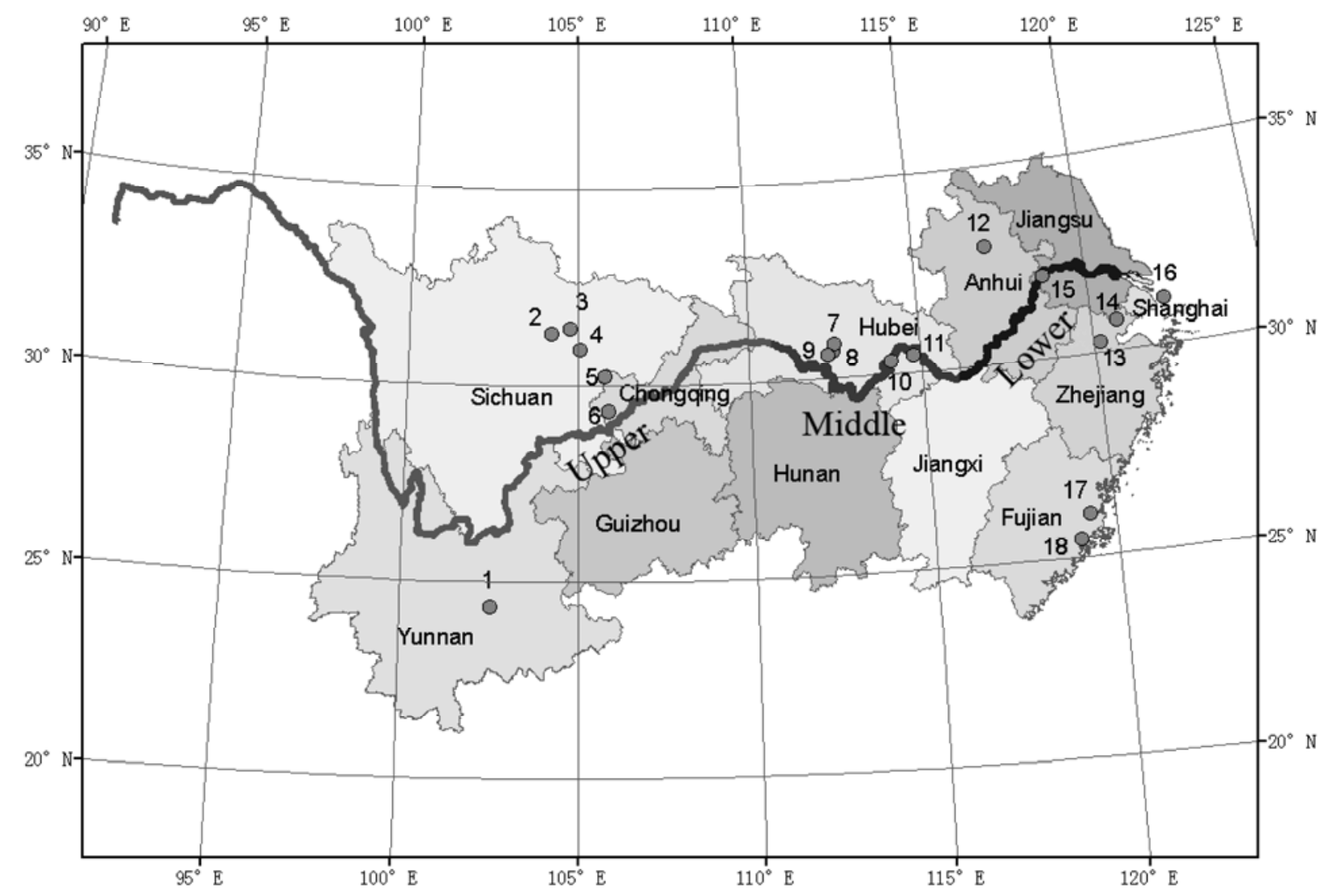

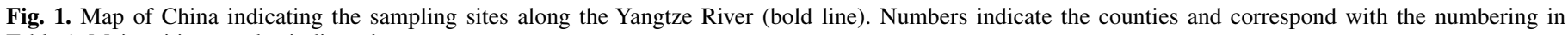
Table 1. Major cities are also indicated. 
$72^{\circ} \mathrm{C}$ for $5 \mathrm{~min}$. Then, the samples were cooled to $4^{\circ} \mathrm{C}$ until recovery. The PCR products were separated in $6 \%$ polyacrylamide denaturing gels. Before sample loading, the PCR products were mixed with equal amounts of loading buffer (Takara) and denatured at $95^{\circ} \mathrm{C}$ for $5 \mathrm{~min}$. After electrophoresis, fragments were visualized using the silver-staining procedure.

Because $F$. asiaticum is homothallic and can also propagate asexually, we applied clone correction to reduce overrepresentation of individual genotypes derived from location-specific clonality (7). Isolates collected from the same sampling site with an identical VNTR profile were considered to be clones and, in these cases, only one representative was taken for the clonecorrected analysis. All clonal isolates, based on sampling site and VNTR markers, also had the same chemotype. Some isolates obtained from different sampling sites with the same VNTR genotype had different chemotypes. Five isolates in Maliang and three isolates in Shayang with an identical VNTR genotype had different chemotypes (15ADON and 3ADON, respectively). Consequently, isolates obtained from different sampling sites with the same genotype were not treated as clones. After clone correction, the total number of isolates was 282 , and the number of isolates before and after clone correction in each sampling site is summarized in Table 1. All further analyses were done with the clone-corrected data.

Data generated from the seven VNTR loci were combined to identify a multilocus genotype $(G)$. Genotype diversity $(G D)$ and multilocus linkage disequilibrium (LD) were estimated by Multi-locus 1.3 (1). GD, the probability that two individual strains taken at random have different genotypes, was calculated by $(n / n-1)\left(1-\Sigma p i^{2}\right)$, where $p i$ is the frequency of the $i$ th genotype and $n$ is the number of individuals sampled (1). The LD measures the nonrandom association of alleles at different gene loci in a population. The test of significance was determined by using 1,000 randomizations in all populations. Gene diversity $(H)$ and Nei's unbiased genetic distance (22) were calculated in the program POPGEN version 1.32 (available at http://www. ualberta.ca/ fyeh/). The correlation analysis between genetic distance and geographical distance of strains from 18 sampling sites was carried out using Mantel test procedure installed in IBD $1.52(3)$.

The analysis of molecular variance (AMOVA) and the determination of pairwise population genetic differentiation $(F s t)$ and gene flow $(\mathrm{Nm})$ were calculated by Arlequin 3.1 (10).
AMOVA was performed to estimate the variance components for the VNTR genotype, partitioning the total genetic diversity into that occurring among and within populations. Pairwise Fst distance estimates were based on the number of different alleles; the statistical significance of pairwise $F s t$ estimates was assessed using a permutation test with 1,000 permutations. Exact tests of population differentiation (15) were conducted using the program GENEPOP 4.0 (27). Analysis of admixture was performed using the Bayesian method implemented in the computer program BAPS 5.2 (8).

Design of chemotype-specific PCR primers. Primers were designed with the aid of the Primer Premier 5 Program (PREMIER Biosoft International, Canada). The trill gene sequences of $13 \mathrm{~B}-\mathrm{FHB}$ strains, including $5 \mathrm{NIV}$ producers (NRRL13383, NRRL13818, NRRL28585, NRRL13393, and NRRL13721), 3 15ADON producers (NRRL6394, NRRL5883, and NRRL26916) and 5 3ADON producers (NRRL6101, NRRL26156, NRRL2903, NRRL28062, and NRRL3288), were compared through multiple sequence alignments. SNPs between strains of different chemotype were translated into PCR amplicons of different lengths (Fig. 2). This allowed designing one set of primers-N11 (5'-CTTGTCAGGCGGCACAGTAG-3'), 15D11 (5'-AAGTATGGTCCAGTTGTCCGTATT-3'), 3D11 (5'-GCAA GTCTGGCGAGGCC- $3^{\prime}$ ), and 11R (5'-TCAAAGGCCAGAGCA ACCC-3') - that generate n 643-bp fragment from NIV-producing strains, a 424-bp fragment from 3ADON producers, and a 342-bp fragment from 15ADON producers, respectively.

Validation of the chemotype-specific PCR assay was performed using 34 strains, including 11 FGSC members and 4 closely related B-trichothecene lineage species ( $F$. lunulosporum, $F$. cerealis, F. culmorum, and F. pseudograminearum) (e-Xtra). Chemotype identities of 17 NRRL strains were published (34), and the mycotoxin chemotypes of the 17 Chinese isolates collected around China were determined by gas chromatography/ mass spectrometry as described by Mirocha et al. (21). According to the size of the amplicons, all of the reference strains were distinguished into different trichothecene chemotypes (Fig. 2). These results were completely congruent with the chemical analyses, indicating the high efficiency and reliability for this multi-PCR detection of three trichothecene chemotypes from BFHB pathogens.

Determination of the $\boldsymbol{F}$. asiaticum chemotype. PCR reactions were carried out in $20-\mu \mathrm{l}$ volumes containing $100 \mathrm{ng}$ of fungal

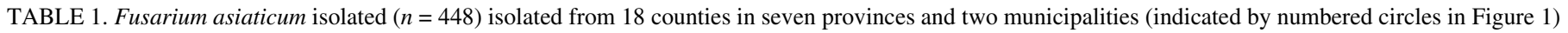

\begin{tabular}{|c|c|c|c|c|c|c|c|}
\hline Region, province $^{\mathrm{a}}$ & Site no. & County & Isolates & Clone-corrected & Latitude & Longitude & Altitude (m) \\
\hline \multicolumn{8}{|l|}{ Upper } \\
\hline Yunnan & 1 & Yuxi & 20 & 2 & $24^{\circ} 21^{\prime}$ & $102^{\circ} 32^{\prime}$ & 1,639 \\
\hline \multirow[t]{3}{*}{ Sichuan } & 2 & Mianzhu & 42 & 30 & $31^{\circ} 19^{\prime}$ & $104^{\circ} 12^{\prime}$ & 593 \\
\hline & 3 & Mianyang & 19 & 11 & $31^{\circ} 28^{\prime}$ & $104^{\circ} 46^{\prime}$ & 453 \\
\hline & 4 & Xinsheng & 45 & 34 & $30^{\circ} 55^{\prime}$ & $105^{\circ} 02^{\prime}$ & 451 \\
\hline \multirow[t]{2}{*}{ Chongqing } & 5 & Tongnan & 9 & 4 & $30^{\circ} 13^{\prime}$ & $105^{\circ} 47^{\prime}$ & 263 \\
\hline & 6 & Yongchuan & 22 & 12 & $29^{\circ} 21^{\prime}$ & $105^{\circ} 53^{\prime}$ & 301 \\
\hline \multicolumn{8}{|l|}{ Middle } \\
\hline \multirow[t]{5}{*}{ Hubei } & 7 & Shayang & 30 & 18 & $30^{\circ} 41^{\prime}$ & $112^{\circ} 28^{\prime}$ & 56 \\
\hline & 8 & Maliang & 29 & 14 & $30^{\circ} 52^{\prime}$ & $112^{\circ} 31^{\prime}$ & 52 \\
\hline & 9 & Jingzhou & 22 & 13 & $30^{\circ} 35^{\prime}$ & $112^{\circ} 19^{\prime}$ & 36 \\
\hline & 10 & Wuchang & 31 & 21 & $30^{\circ} 19^{\prime}$ & $114^{\circ} 07^{\prime}$ & 20 \\
\hline & 11 & Huanggang & 7 & 4 & $30^{\circ} 26^{\prime}$ & $114^{\circ} 47^{\prime}$ & 24 \\
\hline \multicolumn{8}{|l|}{ Lower } \\
\hline Anhui & 12 & Huaiyuan & 24 & 14 & $32^{\circ} 57^{\prime}$ & $117^{\circ} 11^{\prime}$ & 9 \\
\hline \multirow[t]{2}{*}{ Zhejiang } & 13 & Hangzhou & 47 & 33 & $30^{\circ} 15^{\prime}$ & $120^{\circ} 10^{\prime}$ & 17 \\
\hline & 14 & Jiaxing & 27 & 20 & $30^{\circ} 45^{\prime}$ & $120^{\circ} 44^{\prime}$ & 6 \\
\hline Jiangsu & 15 & Nanjing & 16 & 14 & $32^{\circ} 03^{\prime}$ & $118^{\circ} 46^{\prime}$ & 2 \\
\hline Shanghai & 16 & Yongfeng & 30 & 23 & $31^{\circ} 06^{\prime}$ & $121^{\circ} 68^{\prime}$ & 4 \\
\hline \multirow[t]{2}{*}{ Fujian } & 17 & Putian & 6 & 3 & $25^{\circ} 26^{\prime}$ & $119^{\circ} 00^{\prime}$ & 9 \\
\hline & 18 & Fuzhou & 22 & 12 & $26^{\circ} 03^{\prime}$ & $119^{\circ} 18^{\prime}$ & 5 \\
\hline Total & $\ldots$ & $\ldots$ & 448 & 282 & $\ldots$ & $\ldots$ & $\ldots$ \\
\hline
\end{tabular}

${ }^{a}$ Region of Yangtze River and province or municipality. 
DNA. The reaction mixture consisted of $0.25 \mathrm{mM}$ each dATP, dCTP, dGTP, and dTTP and 1 unit of Taq DNA polymerase in $1 \times$ PCR buffer with $1.5 \mathrm{mM} \mathrm{MgCl}_{2}$, with the following primer concentrations: $0.3 \mu \mathrm{M}$ N11, $0.1 \mu \mathrm{M}$ 15D11, $0.15 \mu \mathrm{M}$ 3D11, and $0.3 \mu \mathrm{M} 11 \mathrm{R}$. The cycling conditions consisted of $94^{\circ} \mathrm{C}$ for $3 \mathrm{~min}$; 30 cycles of denaturation $\left(94^{\circ} \mathrm{C}\right)$ for $40 \mathrm{~s}$, annealing $\left(69^{\circ} \mathrm{C}\right)$ for $60 \mathrm{~s}$, and extension $\left(72^{\circ} \mathrm{C}\right)$ for $60 \mathrm{~s}$; followed by a final extension of $72^{\circ} \mathrm{C}$ for $10 \mathrm{~min}$. Then, the samples were cooled to $4^{\circ} \mathrm{C}$ until recovery. PCR products were separated by electrophoresis through $1.5 \%$ agarose gels. Gels were stained with ethidium bromide at $1 \mu \mathrm{g} / \mathrm{ml}$ and photographed under UV light.

\section{RESULTS}

Genetic structure of $\boldsymbol{F}$. asiaticum populations. With the seven VNTR markers, we detected 64 different alleles, and the number of alleles at each locus varied between 5 and 14. Among the 448 $F$. asiaticum isolates analyzed, 256 distinct genotypes were identified. The large number of alleles and genotypes demonstrate a high level of genetic diversity in our Chinese collection.

All strains were classified into three populations according to their geographic origin along the Yangtze River. The upper population contained 54 alleles, while the middle and lower populations had 30 and 41 alleles, respectively ( $e$-Xtra). $G$ (multilocus genotype) was 88,60 , and 108 in upper, middle, and lower populations, respectively. $G D$ values $(0.991,0.981$, and 0.982 , respectively) were high in all populations. Gene diversity $(H)$ of the three populations ranged from 0.49 (lower) and 0.55 (middle)

TABLE 2. Genetic diversity $(G D)$ and multilocus linkage disequilibrium (LD) analysis of Fusarium asiaticum populations from the upper, middle, and lower valley of the Yangtze River

\begin{tabular}{lccccc}
\hline Population & $\mathrm{n}^{\mathrm{a}}$ & $G^{\mathrm{b}}$ & $G D^{\mathrm{c}}$ & $H^{\mathrm{d}}$ & $\mathrm{LD}^{\mathrm{e}}$ \\
\hline Upper & 157 & 88 & 0.991 & 0.62 & $0.092^{* *}$ \\
Middle & 119 & 60 & 0.981 & 0.55 & $0.132^{* *}$ \\
Lower & 172 & 108 & 0.982 & 0.49 & 0.013 \\
Total & 448 & 256 & $\ldots$ & $\ldots$ & $\ldots$ \\
\hline
\end{tabular}

a Sample size $(n)$.

b Number of distinct genotypes $(G)$.

${ }^{c} G D$ within population was calculated as $G D=(n / n-1)\left(1-\Sigma p i^{2}\right)$, where $p i$ is the frequency of the $i$ th genotype, and $n$ is the number of individuals sampled (1).

d Gene diversity $(H)$.

e Measure of multilocus LD; $* *$ indicates significant at $P<0.01$.

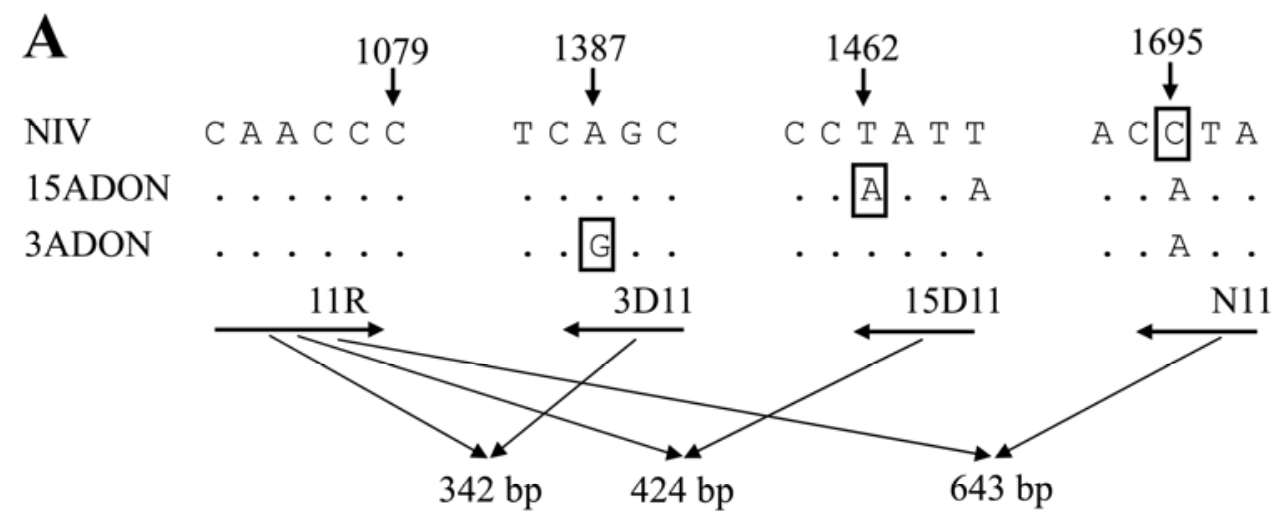

B
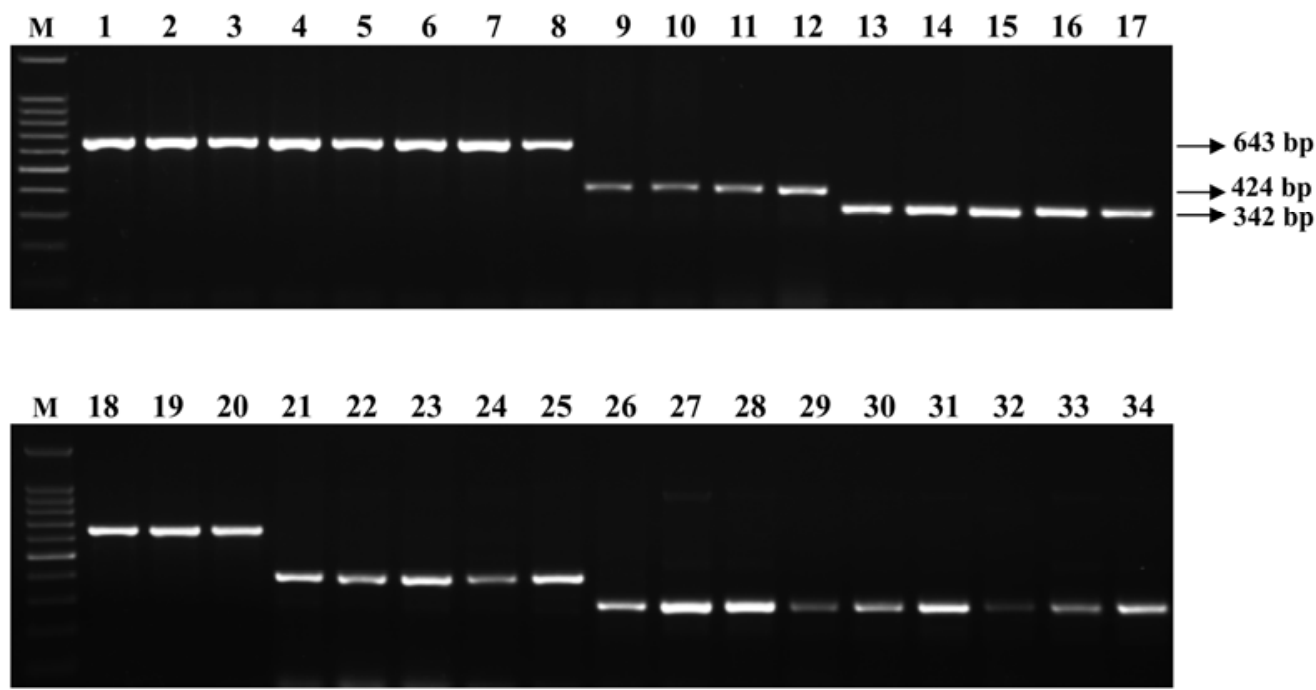

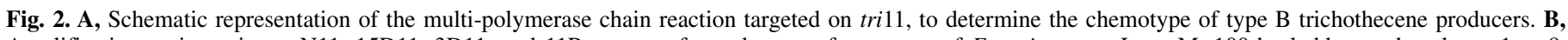

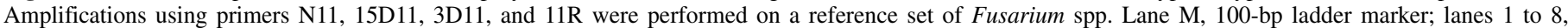

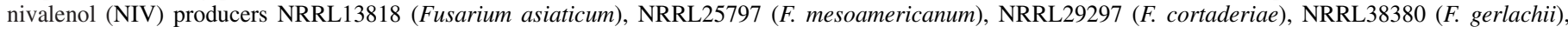

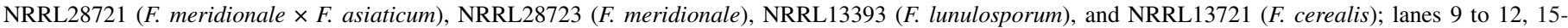

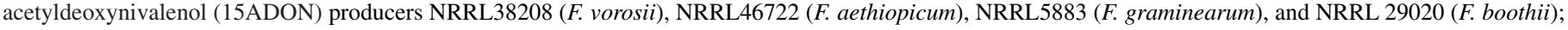

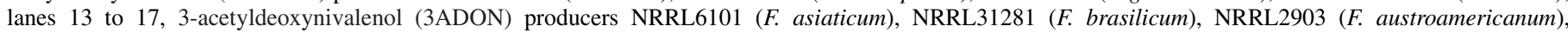

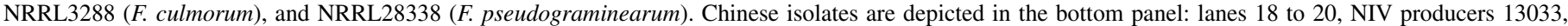

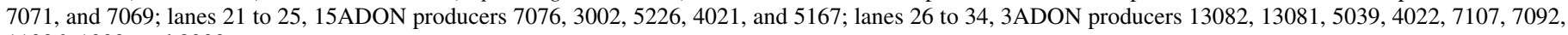
11036, 1009, and 8003 . 
to 0.65 (upper) (Table 2). The LD values were very low in all populations and varied from 0.013 (lower) via 0.092 (upper) to 0.132 (middle) (Table 2). The LD values of upper and middle populations were both significant $(P<0.01)$.

The AMOVA revealed significant genetic variation $(26.8 \%)$ among upper, middle, and lower populations $(F s t=0.247$, $P<0.001$ ), while $73.2 \%$ of the variation was observed among individuals within populations; the overall level of gene flow among populations was low $(N \mathrm{~m}=1.210)$. Pairwise population genetic differentiation $(F s t)$ and gene flow $(N \mathrm{~m})$ across three populations were 0.055 to 0.238 and 1.601 to 8.591 , respectively, and the values of genetic differentiation were all statistically significant $(P<0.001)$. The upper and lower population pair showed the highest Fst and lowest $N \mathrm{~m}$ (Table 3).

More detailed information was obtained at the county level (Table 4). The gene diversity was highest in the mountainous county of Xinsheng in the west $(H=0.592)$ and the lowest in Putian at sea level in the east $(H=0.191)$. The gene diversity of counties in upper reaches was significantly higher than counties in the lower valley ( $t$ test, $P<0.05$ ), indicating higher genetic diversity in mountain regions. The $\mathrm{LD}$ value was highest in Jingzhou $(\mathrm{LD}=0.128)$, and all counties with significant $\mathrm{LD}$ value were in the upper and middle reaches.

Pairwise estimates of genetic distance ( $e$-Xtra) between sampling sites ranged from 0.008 (Hangzhou/Jiaxing) to 0.208

TABLE 3. Pairwise comparisons of gene flow ( $\mathrm{Nm}$ ) (above diagonal) and genetic differentiation (Fst) (below diagonal) among populations of Fusarium asiaticum from the upper, middle, and lower valley of the Yangtze River ${ }^{\mathrm{a}}$

\begin{tabular}{lccc}
\hline Population & Upper & Middle & Lower \\
\hline Upper & $\ldots$ & 1.558 & 1.023 \\
Middle & $0.243^{* *}$ & $\ldots$ & 6.853 \\
Lower & $0.308^{* *}$ & $0.068^{* *}$ & $\ldots$ \\
\hline
\end{tabular}

a Asterisks (**) indicate significant at $P<0.001$.

TABLE 4. Gene diversity and linkage disequilibrium (LD) in all 18 sampling sites

\begin{tabular}{|c|c|c|c|c|}
\hline Region, site & Counties & $H^{\mathrm{b}}$ & $\mathrm{LD}^{\mathrm{c}}$ & $F s t^{\mathrm{d}}$ \\
\hline \multicolumn{5}{|l|}{$\overline{\text { Upper }}$} \\
\hline 1 & Yuxi ${ }^{e}$ & 0.243 & 0.014 & $\ldots$ \\
\hline 2 & Mianzhu & 0.574 & $0.102 * *$ & $\ldots$ \\
\hline 3 & Mianyang & 0.513 & 0.052 & $\begin{array}{l}\cdots \\
\cdots\end{array}$ \\
\hline 4 & Xinsheng & 0.592 & $0.122 * *$ & $0.342 * *$ \\
\hline 5 & Tongnan $^{\mathrm{e}}$ & 0.368 & 0.066 & $\ldots$ \\
\hline 6 & Yongchuan ${ }^{\mathrm{f}}$ & 0.526 & $0.073^{* *}$ & $\ldots$ \\
\hline \multicolumn{5}{|l|}{ Middle } \\
\hline 7 & Shayang ${ }^{\mathrm{f}}$ & 0.485 & $0.128^{* *}$ & $0.471 * *$ \\
\hline 8 & Maliang ${ }^{f}$ & 0.418 & $0.089 * *$ & $\ldots$ \\
\hline 9 & Jingzhou $^{\mathrm{f}}$ & 0.512 & $0.188 * *$ & $0.234 * *$ \\
\hline 10 & Wuchang & 0.476 & 0.054 & $\ldots$ \\
\hline 11 & Huanggang & 0.291 & 0.008 & $\ldots$ \\
\hline \multicolumn{5}{|l|}{ Lower } \\
\hline 12 & Huaiyuan $^{\mathrm{f}}$ & 0.295 & 0.058 & $0.244 * *$ \\
\hline 13 & Hangzhou & 0.376 & 0.052 & $0.225 * *$ \\
\hline 14 & Jiaxing & 0.504 & 0.041 & $0.231 * *$ \\
\hline 15 & Nanjing $f$ & 0.289 & 0.004 & $\ldots$ \\
\hline 16 & Yongfeng & 0.331 & 0.012 & $0.141 * *$ \\
\hline 17 & Putian ${ }^{\mathrm{e}}$ & 0.191 & 0.003 & \\
\hline 18 & Fuzhou $^{\mathrm{f}}$ & 0.307 & 0.016 & $0.387^{* *}$ \\
\hline
\end{tabular}

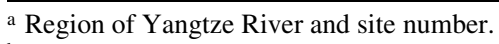

b Gene diversity $(H)$.

c Asterisks (**) indicate significant at $P<0.001$.

${ }^{\mathrm{d}}$ Genetic differentiation ( $F$ st) was calculated between nivalenol (NIV) and 3acetyldeoxynivalenol (3ADON) populations in seven co-occurred counties, except Fuzhou. The Fst of Fuzhou was calculated between 15-acetyldeoxynivalenol and 3ADON populations.

${ }^{\text {e }}$ Fst and LD of this sampling site were based on a very low number of genotypes ( $<5$ genotypes). This data is for reference only.

${ }^{\mathrm{f}}$ Fst and LD of this sampling site were based on $<20$ genotypes. This data is for reference only.
(Yuxi/Putian). Based on the matrix, the Mantel test revealed that there was a highly significant positive correlation $(r=0.5147, P<$ 0.001 ) between estimates of genetic distances (22) and geographical distances between populations (Fig. 3). Significance was assessed by 1,000 randomizations.

Chemotype diversity. A PCR assay based on the trill gene was conducted to determine the chemotype of each $F$. asiaticum isolate. The assay indicated that NIV, 3ADON, and 15ADON producers were present among the 282 clone-corrected isolates tested; 109 isolates yielded a NIV-specific PCR fragment, 159 isolates had a $3 \mathrm{ADON}$-specific fragment, and a small set $(n=14)$ of strains produced a 15ADON-specific fragment. The geographical distribution of chemotypes showed a dramatic gradient; the NIV chemotype was remarkably more frequent in upper valleys and the $3 \mathrm{ADON}$ chemotype was predominant in the middle and lower reaches of the Yangtze River (Fig. 4). In all, $>86 \%$ of strains collected from upper valleys of the Yangtze River were NIV producers, while $79 \%$ isolates obtained from middle and lower valleys were DON producers (3ADON and $15 \mathrm{ADON}$ ). $3 \mathrm{ADON}$ producers were predominant in the middle and lower valleys of the Yangtze River but not in Fujian Province. The number of 15ADON producers as a percentage of the total DON producers was high in Fujian (Fig. 4).

In order to evaluate the genetic variation of different populations in different areas along the Yangtze River, samples were divided into nine groups based on geographic origin and trichothecene chemotype. These groups consisted of 3ADON, $15 \mathrm{ADON}$, and NIV producers from upper reaches (3U, 15U, and $\mathrm{NU})$; $3 \mathrm{ADON}, 15 \mathrm{ADON}$, and NIV producers from middle reaches (3M, 15M, and NM); and 3ADON, 15ADON, and NIV producers from lower reaches (3L, 15L, and NL). Pairwise Fst values showed significant differentiation between NIV and 3ADON populations (NU-3M, Fst $=0.257, P<0.001$; NU-3L, $F s t=0.312, P<0.001)$ which were predominant in different areas. A small but statistically significant degree of genetic differentiation was also observed between $3 \mathrm{ADON}$ populations from middle and lower reaches $(3 \mathrm{M}-3 \mathrm{~L}, F s t=0.058, P<0.001)$. Fst values among populations within the upper (NU-3U, $F s t=0.282$, $P<0.001$; NU-15U, Fst $=0.269, P<0.001)$, middle $(3 \mathrm{M}-15 \mathrm{M}$, $F s t=0.211, P<0.001 ; 3 \mathrm{M}-\mathrm{NM}, F s t=0.248, P<0.001)$, and

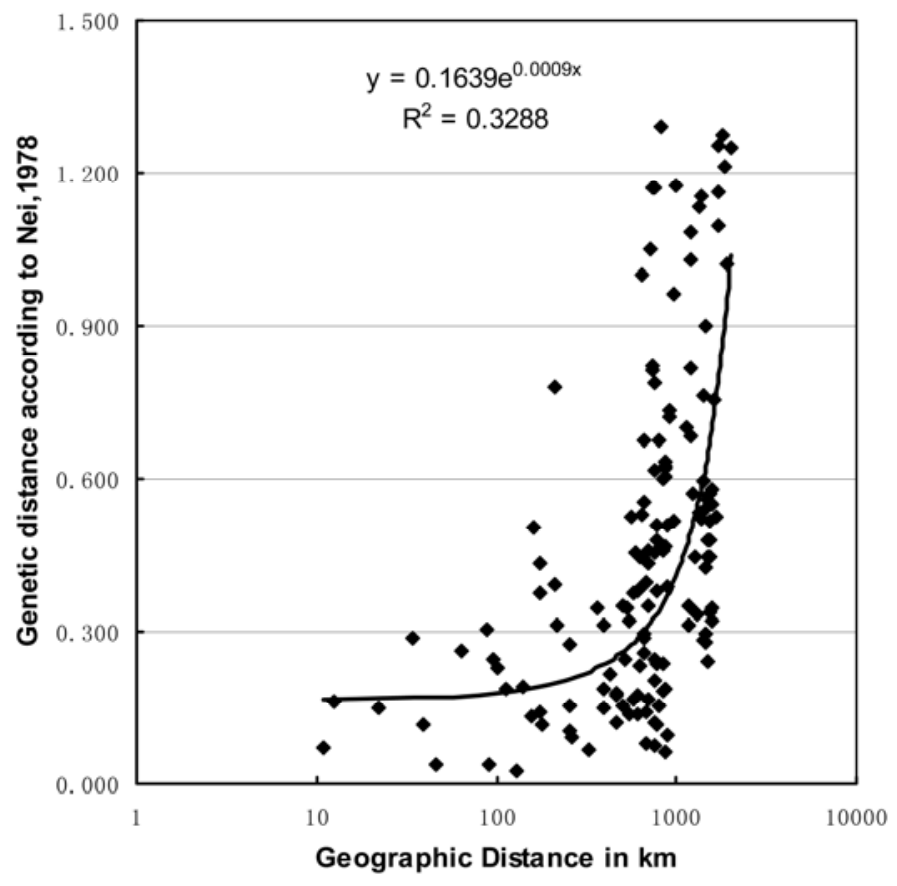

Fig. 3. Correlation between calculated genetic distance and geographic distance among 18 sampling points $(r=0.5808, P<0.001)$. 
lower (3L-15L, Fst $=0.291, P<0.001 ; 3 \mathrm{~L}-\mathrm{NL}, F s t=0.194, P<$ $0.001)$ reaches were all high. The $\mathrm{LD}$ value of $\mathrm{NU}, 3 \mathrm{M}$, and $3 \mathrm{~L}$ was $0.048(\mathrm{NU}), 0.078(3 \mathrm{M})$, and $0.008(3 \mathrm{~L})$, which was lower than the value of populations including the whole isolates in each area (upper, middle, and lower reaches), respectively (Table 2).

A Bayesian clustering method was used to investigate admixture between the three dominant populations (NU, 3M, and 3L) in each area. Admixture probabilities $>95 \%$ were obtained for 24 of the 227 isolates genotyped (Table 5), and analyses of population structure after removal of the 24 admixed isolates produced results similar to those described above; there was also significant pairwise $F s t$ (Table 6) and a similar LD value (NU $=0.045,3 \mathrm{M}=$ 0.069 , and $3 \mathrm{~L}=0.009)$. We found that 9 of the $80(11.3 \%) \mathrm{NIV}$ strains had significant admixture probabilities $(P<0.001)$. Among these nine strains, Bayesian posterior mean estimates for the proportion of the genome derived from the $3 \mathrm{M}$ or $3 \mathrm{~L}$ populations was 44 to $98 \%$ (Table 5), and the proportion from $3 \mathrm{M}$ was significantly $(P<0.005)$ more than from 3L. Only 3 of 147 $(2.0 \%) 3 \mathrm{ADON}$ strains had significant $(P<0.001)$ admixture probabilities with some proportion of their genomes derived from the NU population. NIV isolates with $3 \mathrm{ADON}$ genetic material were significantly (exact test; $P<0.001$ ) more common than 3ADON isolates with NIV genetic material. The remaining admixed isolates were composed of genetic material from the $3 \mathrm{M}$ and $3 \mathrm{~L}$ populations, which likely share a high level of background ground relatedness given their close genetic relationship $(F s t=$ 0.054 when all isolates are included in the analysis).

In order to get more detailed information about the genetic variation and potential migrants of the pathogen, population genetic differentiation $(F s t)$ between 3ADON and NIV producers and multilocus LD in the same sampling site were estimated. This analysis focused on seven counties where 3ADON and NIV producing isolates co-occurred. The result revealed significant genetic differentiation $(P<0.001)$ between the two chemotype populations existing in each sampling site; $F$ st was from 0.141 (Yongfeng) to 0.471 (Shayang) and the LD value of Xinsheng, Shayang, and Jingzhou populations was significant $(P<0.001)$, although it was low in other four low valley counties (Table 4). This result showed a trend that the genetic differentiation and multilocus LD increased from east to west along the Yangtze River. We also found that, in each region of the Yangtze River, the LD of counties was relatively high in counties where NIV and $3 \mathrm{ADON}$ producers co-occurred, indicating that the differences between NIV and 3ADON populations contribute significantly to the LD in the same sampling site.

\section{DISCUSSION}

The low LD estimates detected in all counties suggest that frequent outcrossing occurred in all three populations along the Yangtze River. This is in agreement with the previous reports on other members of the FGSC such as $F$. graminearum of which low LD values were found in populations in the United States $(28,40)$. However, no study until now could present a direct evidence of sexual mating or crossing in the field. In contrast, clear LD was observed in a single interbreeding population of $F$. graminearum collected from wheat in Zhenjiang province using restriction fragment length polymorphism (RFLP) markers (12). We found no significant LD values for Zhejiang whereas Gale et al. (12) did observe significant LD values. This revealed that the original high LD found may have been decreased over time (2000 to 2005) due to outcrossing. We assume that local significant LD

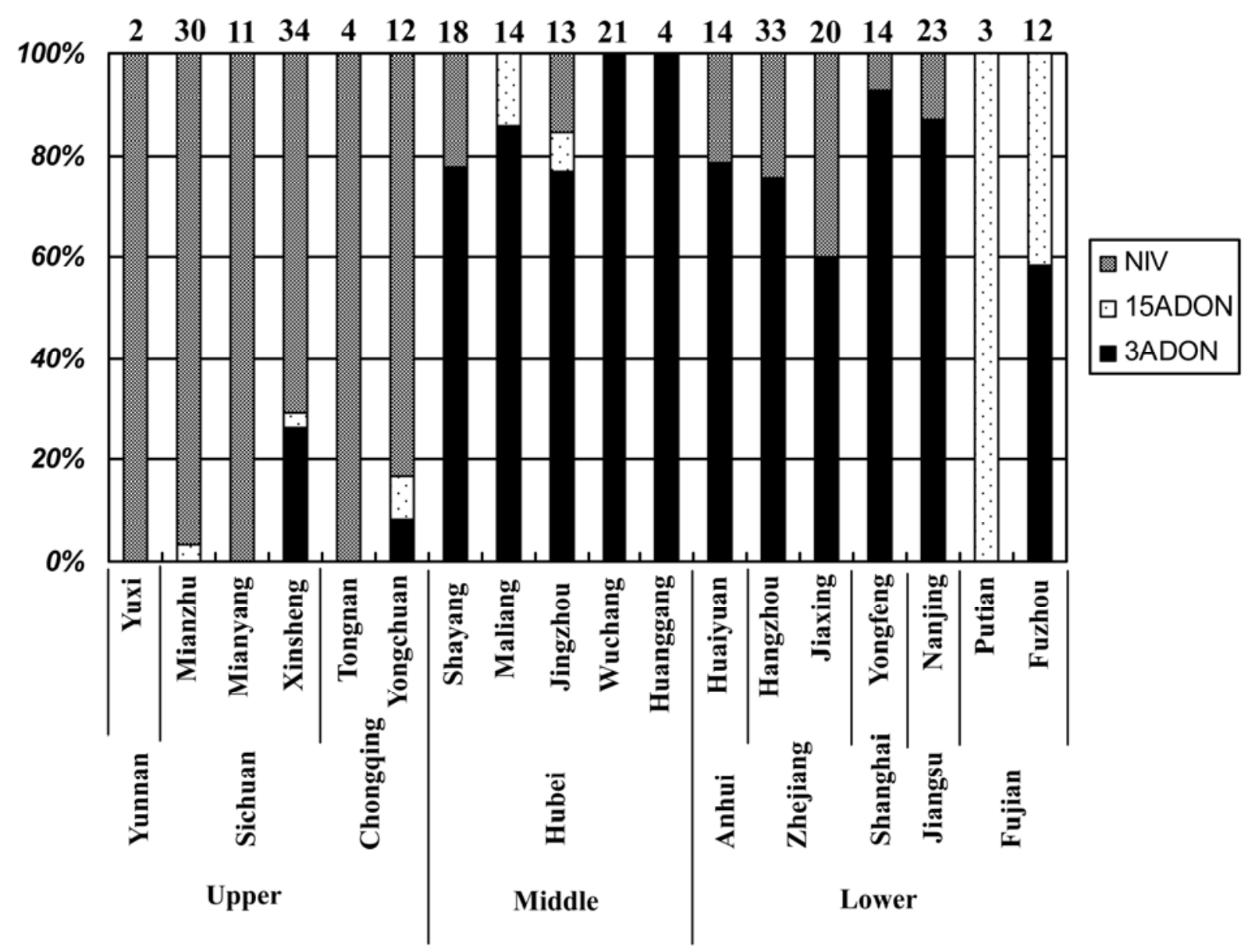

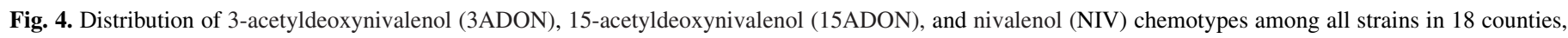
which are arranged from the upper valleys to the lower valleys of the Yangtze River. Numbers at the top indicated the number of isolates in each county. 
values indicate continuous gene flow from migrants, resulting in local gametic disequilibria discussed in more detail below. When these migrants are absorbed in the gene pool, the $\mathrm{LD}$ values are reduced, and this may explain the differences in LD values found over time and space in the different Fusarium populations.

AMOVA showed that genetic variation within the upper, middle, and lower populations was higher than among these populations, which may be due to the prevalence of sexual reproduction, population size, or high levels of migration. Previous studies $(5,11,12)$ have found similar results for $F$. graminearum populations from wheat, and sexual recombination in the field may result in the formation of novel genotypes (4). The results of the Mantel test suggested restricted gene flow among sampling sites and were at least partly consistent with the model of isolation by distance (37). Also, Zeller et al. (40) found significant negative correlations between population identity and distance of different Gibberella zeae populations in the United States. In our case, the Mantel test revealed significant correlation $(r=0.5147$, $P<0.001)$ between genetic distances and geographical distances. Previously, Zeller et al. reported a significant negative correlation $(r=-0.591, P<0.001)$. It is clear that these correlations will only be shallow, if detected at all, because they do not take other factors that are important for migration into account, such as geographical barriers or prevailing winds. Moreover, Dusabenyagasani et al. (9) also performed RAPD genotyping on $72 \mathrm{~F}$. graminearum strains collected from Canada in 1999, and found very little population differentiation with distance. The genetic variation among the upper, middle, and lower populations was significant $(P<0.001)$, although its value is lower. It demonstrated that these regions contain three different populations, with limited genetic exchange among populations. We also observed that $F s t$ between upper and middle populations was much higher than between middle and lower populations. This phenomenon reflected the presence of geographic barriers such as high mountain ridges adding to the isolation by distance as discussed above.

In previous studies of these samples, we have found that the geographical distribution of chemotypes showed a dramatic

TABLE 5. Isolates with significant admixture probabilities from Bayesian analysis $^{\mathrm{a}}$

\begin{tabular}{lcccc}
\hline & & \multicolumn{3}{c}{ Percent genome represented by each population } \\
\cline { 3 - 5 } No. & Source population & NU & $3 \mathrm{M}$ & $3 \mathrm{~L}$ \\
\hline 05023 & NU & 0.15 & 0.84 & 0.01 \\
05034 & NU & 0.45 & 0.55 & 0.00 \\
05035 & NU & 0.33 & 0.62 & 0.05 \\
05048 & NU & 0.45 & 0.55 & 0.00 \\
05061 & NU & 0.33 & 0.00 & 0.67 \\
05063 & NU & 0.06 & 0.01 & 0.93 \\
05070 & NU & 0.22 & 0.72 & 0.06 \\
05081 & NU & 0.56 & 0.44 & 0.00 \\
05084 & NU & 0.02 & 0.24 & 0.74 \\
05105 & 3M & 0.67 & 0.33 & 0.00 \\
05108 & 3M & 0.00 & 0.54 & 0.46 \\
05113 & 3M & 0.48 & 0.52 & 0.00 \\
05115 & 3M & 0.00 & 0.26 & 0.74 \\
05122 & 3M & 0.00 & 0.40 & 0.60 \\
05135 & 3M & 0.06 & 0.16 & 0.78 \\
05142 & 3M & 0.00 & 0.00 & 1.00 \\
05145 & 3M & 0.00 & 0.45 & 0.55 \\
05172 & 3M & 0.00 & 0.41 & 0.59 \\
05173 & 3L & 0.00 & 0.65 & 0.35 \\
05198 & 3L & 0.00 & 0.90 & 0.10 \\
05205 & 3L & 0.08 & 0.65 & 0.35 \\
05206 & 3L & 0.00 & 1.00 & 0.00 \\
05212 & 3L & 0.00 & 0.48 & 0.52 \\
05245 & 3L & 0.00 & 0.81 & 0.19 \\
\hline
\end{tabular}

${ }^{\mathrm{a}} \mathrm{NU}=$ nivalenol population from the upper reaches of the Yangtze River, $3 \mathrm{M}=3$-acetyldeoxynivalenol ( $3 \mathrm{ADON})$ population from the middle reaches, and $3 \mathrm{~L}=3 \mathrm{ADON}$ population from the lower reaches. gradient along the Yangtze River and we hypothesized that isolates from the mountain-rich provinces represented the old FHB population and that NIV-producing isolates may have dominated Chinese FHB populations in the past but are currently being replaced by DON producers (39). In this study, we tested this hypothesis by combining genotyping analysis using "neutral" VNTR markers with the chemotype of the isolates.

After testing $448 F$. asiaticum isolates with the newly designed chemotype-specific primers, the DON producers were further differentiated into $3 \mathrm{ADON}$ or $15 \mathrm{ADON}$ chemotypes. This demonstrates that $3 \mathrm{ADON}$ and not $15 \mathrm{ADON}$ predominates in the middle and lower valleys of the Yangtze River. This geographic gradient in trichothecene chemotype frequency was similar to the recently reported shift in the $F$. graminearum population in North American. Ward et al. (35) observed a pronounced longitudinal cline of the trichothecene chemotype frequency distribution in Canada, in which $3 \mathrm{ADON}$ producers were most frequent in eastern Canada and decreased in frequency in each province sampled from east to west across Canada. They also documented a recent and significant shift in FHB pathogen composition by demonstrating that the $3 \mathrm{ADON}$ chemotype frequency in western Canada increased >14-fold between 1998 and 2004. The change of 3ADON chemotype frequency was also observed in China. Zhang et al. (41) reported the trichothecene chemotypes of $F$. graminearum SCAR group 5 (now called $F$. asiaticum) collected in 1999 from China. In their study, 53.1\% of all isolates obtained from the middle valley of the Yangtze River were $3 \mathrm{ADON}$ producers. However, in the present study, we observed a ratio of $3 \mathrm{ADON}$ producers in the middle valley of $87.1 \%$. This indicated that the $3 \mathrm{ADON}$ producers increased significantly (exact test, $P<$ 0.001 ) in the middle reaches in the 6 years between 1999 and 2005.

We also tested the direction of the gene flow using Bayesian analysis. Pairwise Fst distance values showed significant differentiation between NIV and 3ADON groups (NU-3M, Fst $=$ $0.227, P<0.001$; NU-3L, Fst $=0.256, P<0.001$ ), indicating that groups defined based on trichothecene chemotype represented distinct populations, even where isolates from these groups cooccur (Table 4). Bayesian analysis revealed a biased gene flow from $3 \mathrm{ADON}$ populations in the middle and lower reaches to the NIV population in the upper valley, which may determine the migration direction of $F$. asiaticum. 3ADON isolates from middle and lower reaches showed a close genetic relationship $(F s t=$ 0.053 ), which is likely due to recent migration of $3 \mathrm{ADON}$ isolates. To further verify this conjecture, we performed LD analysis. The LD value of populations including $3 \mathrm{ADON}, \mathrm{NIV}$, and 15 ADON isolates in each area were all higher than the dominant chemotype populations (NU, 3M, and $3 \mathrm{~L}$, respectively), indicating there has been insufficient time for random mating to break down LD created by migrants. The situation of counties where different chemotype producers co-occurred provided more detailed evidence about it. Both the genetic differentiation between 3ADON- and 15ADON-producing isolates and multilocus LD increased from east to west along the Yangtze River. Consistent with the 3ADON isolates migrating from east to west, the genetic differentiation and LD was larger in the west. In addition, all counties with significant LD value belonging to

TABLE 6. Pairwise genetic differentiation (above diagonal) and permutation test probability (below diagonal) estimates with admixed isolates excluded

\begin{tabular}{lccc}
\hline Population $^{\mathrm{a}}$ & $\mathrm{NU}$ & $3 \mathrm{M}$ & $3 \mathrm{~L}$ \\
\hline $\mathrm{NU}$ & $\ldots$ & 0.343 & 0.339 \\
$3 \mathrm{M}$ & $<0.001$ & $\ldots$ & 0.104 \\
$3 \mathrm{~L}$ & $<0.001$ & $<0.001$ & $\ldots$ \\
\hline
\end{tabular}

${ }^{\text {a }} \mathrm{NU}=$ nivalenol population from the upper reaches of the Yangtze River, $3 \mathrm{M}=3$-acetyldeoxynivalenol ( $3 \mathrm{ADON})$ population from the middle reaches, and $3 \mathrm{~L}=3 \mathrm{ADON}$ population from the lower reaches. 
upper and middle reaches provided additional evidence of this. Based on (i) the cline in trichothecene chemotypes, (ii) a recent and significant shift of $3 \mathrm{ADON}$ producers in FHB pathogen composition in the middle valley, (iii) the bias gene flow from $3 \mathrm{ADON}$ to NIV populations, and (iv) the substantial genetic differentiation and LD between sympatric NIV and 3ADON populations along the Yangtze River, we conclude that a 3ADON population from eastern China has only recently spread west. This spread may have caused the increased problems of FHB on barely and wheat observed in the last decade. Ward et al. (35) reported that $F$. graminearum isolates from $3 \mathrm{ADON}$ populations produced significantly more trichothecene and had significantly higher fecundity and growth rates. This may also be true for $F$. asiaticum, and this selective advantage may have driven the change to a pathogen population which is more toxigenic and potentially more vigorous. To further validate and explain the hypothesis, monitoring the shifts of genetic variation and trichothecene chemotype composition of the pathogen along the Yangtze River and detecting the biological characteristics of different populations are necessary in the future.

This study showed that $F$. asiaticum isolates that produce 3ADON are spreading from the lower and central valleys of the Yangtze River. Although this spread may be delayed by physical barriers such as mountain ridges, we expect that the driving force articulated by the capacity to produce different type B trichothecenes will cause a further spread of $3 \mathrm{ADON}$ producers in the future. These results have important implications for the control of scab and the reduction of trichothecene contamination of cereals in southern China. Our findings highlight the potential for the exchange of adaptations between FHB populations via recombination and emphasize the need to consider pathogen variation in addition to host variation in FHB management. Specifically, the pathogen variation in the severe disease epidemic area of China is required to be considered in the development of cereal cultivars with broad-based resistance to the pathogens.

\section{ACKNOWLEDGMENTS}

This work was supported by the National Basic Research and Development Program (973 Program) (no. 2009CB119200), a project of the Special Project of Commonweal Industry Scientific Research (nyhyzx07-048), and a project of International Scientific and Technical Cooperation (No. 2009DFA31980). We thank T. J. Ward (United States Department of Agriculture [USDA]) for revising this article and suggestions for the data analysis, and K. O'Donnell (USDA) for the NRRL isolates used as the reference set.

\section{LITERATURE CITED}

1. Agapow, P. M., and Burt, A. 2001. Indices of multilocus linkage disequilibrium. Mol. Ecol. Notes 1:101-102.

2. Alexander, N. J., Hohn, T. M., and McCormick, S. P. 1998. The TRI11 gene of Fusarium sporotrichioides encodes a cytochrome P-450 monooxygenase required for $\mathrm{C}-15$ hydroxylation in trichothecene biosynthesis. Appl. Environ. Microbiol. 64:221-225.

3. Bohonak, A. J. 2002. IBD (isolation by distance): A program for analyses of isolation by distance. J. Hered. 93:153-154.

4. Bowden, R. L., and Leslie, J. F. 1999. Sexual recombination in Gibberella zeae. Phytopathology 89:182-188.

5. Burlakoti, R. R., Ali, S., Secor, G. A., Neate, S. M., McMullen, M. P., and Adhikari, T. B. 2008. Genetic relationships among populations of Gibberella zeae from barley, wheat, potato, and sugar beet in the upper Midwest of the United States. Phytopathology 98:969-976.

6. Chandler, E. A., Simpson, D. R., Thomsett, M. A., and Nicholson, P. 2003. Development of PCR assays to Tri7 and Tril3 and characterisation of chemotypes of Fusarium graminearum, Fusarium culmorum, and Fusarium cerealis. Physiol. Mol. Plant Pathol. 62:355-367.

7. Chen, R. S., Boeger, J. M., and McDonald, B. A. 1994. Genetic stability in a population of a plant pathogenic fungus over time. Mol. Ecol. 3:209-218.

8. Corander, J., and Marttinen, P. 2006. Bayesian identification of admixture events using multilocus molecular markers. Mol. Ecol. 15:2833-2843.
9. Dusabenyagasani, M., Dostaler, D., and Hamelin, R. C. 1999. Genetic diversity among Fusarium graminearum strains from Ontario and Quebec. Can. J. Plant Pathol. 21:308-314.

10. Excoffier, L., Laval, G., and Schneider, S. 2005. ARLEQUIN ver. 3.0: an integrated software package for population genetics data analysis. Evol. Bioinform. 1:47-50.

11. Fernando, W. G. D., Zhang, J. X., Dusabenyagasani, M., Guo, X. W., Ahmed, H., and McCallum, B. 2006. Genetic diversity of Gibberella zeae isolates from Manitoba. Plant Dis. 90:1337-1342.

12. Gale, L. R., Chen, L. F., Hernick, C. A., Takamura, K., and Kistler, H. C. 2002. Population Analysis of Fusarium graminearum from wheat fields in eastern China. Phytopathology 92:1315-1322.

13. Giraud, T., Fournier, E., Vautrin, D., Solignac, M., Vercken, E., Bakan, B., and Brygoo, Y. 2002. Isolation of eight polymorphic microsatellite loci, using an enrichment protocol, in the phytopathogenic fungus Fusarium culmorum. Mol. Ecol. Notes 2:121-123.

14. Goswami, R. S., and Kistler, H. C. 2004. Heading for disaster: Fusarium graminearum on cereal crops. Mol. Plant Pathol. 5:515-525.

15. Goudet, J., Raymond, M., de Meeus, T., and Rousset, F. 1996. Testing differentiation in diploid populations. Genetics 144:1933-1940.

16. Jennings, P., Coates, M. E., Turner, J. A., Chandler, E. A., and Nicholson, P. 2004. Determination of deoxynivalenol and nivalenol chemotypes of Fusarium culmorum isolates from England and Wales by PCR assay. Plant Pathol. 53:182-190.

17. Kimura, M., Kaneko, I., Komiyama, M., Takatsuki, A., Koshino, H., Yoneyama, K., and Yamaguchi, I. 1998. Trichothecene 3-O-acetyltransferase protects both the producing organism and transformed yeast from related mycotoxins. Cloning and characterization of Tri101. J. Biol. Chem. 273:1654-1661.

18. Maier, F. J., Miedaner, T., Hadeler, B., Felk, A., Salomon, S., Lemmens, M., Kassner, H., and Schäfer, W. 2006. Involvement of trichothecenes in Fusarioses of wheat, barley and maize evaluated by gene disruption of the trichodiene synthase (Tri5) gene in three field isolates of different chemotype and virulence. Mol. Plant Pathol. 7:449-461.

19. Miller, J. D., and Greenhalgh, R. 1991. Trichothecene chemotypes of three Fusarium species. Mycologia 83:121-130.

20. Mirocha, C. J., Abbas, H. K., Windels, C. E., and Xie, W. 1989. Variation in deoxynivalenol, 15-acetyldeoxynivalenol, 3-acetyldeoxynivalenol, and zearalenone production by Fusarium graminearum isolates. Appl. Environ. Microbiol. 55:1315-1316.

21. Mirocha, C. J., Kolaczkowski, E., Xie, W. P., Yu, H., and Jelen, H. 1998. Analysis of deoxynivalenol and its derivatives (batch and single kernel) using gas chromatography/mass spectrometry. J. Agric. Food Chem. 46:1414-1418.

22. Nei, M. 1978. Estimation of average heterozygosity and genetic distance from a small number of individuals. Genetics 89:583-590.

23. O’Donnell, K., Kistler, H. C., Tacke, B. K., and Casper, H. H. 2000. Gene genealogies reveal global phylogeographic structure and reproductive isolation among lineages of Fusarium graminearum, the fungus causing wheat scab. Proc. Natl. Acad. Sci. USA 97:7905-7910.

24. O’Donnell, K., Ward, T. J., Aberra, D., Kistler, H. C., Aoki, T., Orwig, N., Kimura, M., Bjornstad, S., and Klemsdal, S. S. 2008. Multilocus genotyping and molecular phylogenetics resolve a novel head blight pathogen within the Fusarium graminearum species complex from Ethiopia. Fungal Genet. Biol. 45:1514-1522.

25. O'Donnell, K., Ward, T. J., Geiser, D. M., Corby Kistler, H., and Aoki, T. 2004. Genealogical concordance between the mating type locus and seven other nuclear genes supports formal recognition of nine phylogenetically distinct species within the Fusarium graminearum clade. Fungal Genet. Biol. 41:600-623.

26. Qu, B., Li, H. P., Zhang, J. B., Xu, Y. B., Huang, T., Wu, A. B., Zhao, C. S., Carter, J., Nicholson, P., and Liao, Y. C. 2008. Geographic distribution and genetic diversity of Fusarium graminearum and F. asiaticum on wheat spikes throughout China. Plant Pathol. 57:15-24.

27. Raymond, M., and Rouset, F. 2005. GENEPOP (version 1.2). Population genetics software for exact tests and ecumenicism. J. Hered. 86:248-249.

28. Schmale Iii, D. G., Leslie, J. F., Zeller, K. A., Saleh, A. A., Shields, E. J., and Bergstrom, G. C. 2006. Genetic structure of atmospheric populations of Gibberella zeae. Phytopathology 96:1021-1026.

29. Starkey, D. E., Ward, T. J., Aoki, T., Gale, L. R., Kistler, H. C., Geiser, D. M., Suga, H., Toth, B., Varga, J., and O’Donnell, K. 2007. Global molecular surveillance reveals novel Fusarium head blight species and trichothecene toxin diversity. Fungal Genet. Biol. 44:1191-1204.

30. Suga, H., Gale, L. R., and Kistler, H. C. 2004. Development of VNTR markers for two Fusarium graminearum clade species. Mol. Ecol. 4:468470.

31. Ueno, Y., Nakajima, M., Sakai, K., Ishii, K., and Sato, N. 1973. Comparative toxicology of trichothec mycotoxins: inhibition of protein synthesis in animal cells. J. Biochem. (Tokyo) 74:285-296.

32. Vogelgsang, S., Widmer, F., Jenny, E., and Enkerli, J. 2009. Characteri- 
sation of novel Fusarium graminearum microsatellite markers in different Fusarium species from various countries. Eur. J. Plant Pathol. 123:477482.

33. Waalwijk, C., Kastelein, P., Vries, I., Kerenyi, Z., Lee, T., Hesselink, T., Kohl, J., and Kema, G. 2003. Major changes in Fusarium spp. in wheat in the Netherlands. Eur. J. Plant Pathol. 109:743-754.

34. Ward, T. J., Bielawski, J. P., Kistler, H. C., Sullivan, E., and O'Donnell, K. 2002. Ancestral polymorphism and adaptive evolution in the trichothecene mycotoxin gene cluster of phytopathogenic Fusarium. Proc. Natl. Acad. Sci. USA 99:9278-9283.

35. Ward, T. J., Clear, R. M., Rooney, A. P., O’Donnell, K., Gaba, D., Patrick, S., Starkey, D. E., Gilbert, J., Geiser, D. M., and Nowicki, T. W. 2008. An adaptive evolutionary shift in Fusarium head blight pathogen populations is driving the rapid spread of more toxigenic Fusarium graminearum in North America. Fungal Genet. Biol. 45:473-484

36. Windels, C. E. 2000. Economic and social impacts of Fusarium head blight: changing farms and rural communities in the northern great plains. Phytopathology 90:17-21.

37. Wright, S. 1943. Isolation by distance. Genetics 28:114-138.

38. Xu, Y. G., and Chen, L. F. 1993. Wheat Scab: Theory and Practice on Control. Jiangsu Science and Technology Publishing House, Nanjing, China.

39. Yang, L., van der Lee, T., Yang, X., Yu, D., and Waalwijk, C. 2008. Fusarium populations on Chinese barley show a dramatic gradient in mycotoxin profiles. Phytopathology 98:719-727.

40. Zeller, K. A., Bowden, R. L., and Leslie, J. F. 2004. Population differentiation and recombination in wheat scab populations of Gibberella zeae from the United States. Mol. Ecol. 13:563-571.

41. Zhang, J. B., Li, H. P., Dang, F. J., Qu, B., Xu, Y. B., Zhao, C. S., and Liao, Y. C. 2007. Determination of the trichothecene mycotoxin chemotypes and associated geographical distribution and phylogenetic species of the Fusarium graminearum clade from China. Mycol. Res. 111:967-975. 